\title{
Eosinophilic Cystitis in a 7-Year-Old-Boy with Eosinophilic Esophagitis, Asthma, Food Allergies, and Atopic Dermatitis
}

Russell J. Hopp ${ }^{1^{*}}$ and John M. Donovan ${ }^{2}$

${ }^{1}$ Department of Pediatrics and Medicine, Creighton University, USA

${ }^{2}$ Children's Hospital and Medical Center, Omaha, NE, USA

*Corresponding author: Russell J. Hopp, Department of Pediatrics and Medicine, Creighton University, Omaha, Nebraska, USA, E-mail: rhopp@creighton.edu

Received date: March 17, 2017; Accepted date: March 23, 2017; Published date: March 31, 2017

Copyright: (c) 2017 Hopp RJ, et al. This is an open-access article distributed under the terms of the Creative Commons Attribution License, which permits unrestricted use, distribution, and reproduction in any medium, provided the original author and source are credited.

\section{Introduction}

World-wide, allergic diseases, all of which are associated with blood and tissue eosinophilia, are increasing. In the past two decades, a relatively new allergic disease, eosinophilic esophagitis (EoE), has become a common occurrence in pediatric aged children, and is often a new diagnosis in a child with another allergic disease [1]. In contrast, eosinophilic cystitis (EC), remains a rare eosinophilic-mediated disease [2]. There continues to be debate as to the etiology of the disorder. We report a child with new onset EC, who had 4 other preexistent allergic diseases.

\section{Case Report}

A 7 year-old Caucasian male developed eosinophilic cystitis, with previous diagnoses of atopic dermatitis (AD), asthma, food allergy, and eosinophilic esophagitis (EoE). At the time of his initial presentation to our referral academic Eosinophilic Esophagitis Clinic (age 4 years), he had an existing diagnosis of EoE and atopic dermatitis, with an extensive list of positive ImmunoCAP RAST positive tests, all of which were being avoided to treat both his EoE and $\mathrm{AD}$, or for primary food allergy concerns. Due to the difficulty in appropriate nutritional support, he was placed on swallowed Budesonide, and a separate course of topical AD therapy was initiated. A repeat esophageal biopsy 8 weeks afterwards was normal, and he maintained on a daily swallowed Budesonide dose. Over the ensuing 3 years, he developed asthma, documented with a $20 \%$ improvement in forced vital capacity at one-second and stable pulmonary history after the initiation of inhaled fluticasone diproprionate (88 micrograms, inhaled BID). Foods with marginal positive ImmunoCAP RAST values, without a clinical history, had been slowly re-added, but nothing new had been added for one year before the onset of hematuria. At the time of the onset of hematuria and biopsy he was avoiding: milk, eggs, fish peanuts, tree nuts, bananas, melons, dates, fig, flaxseed, green beans, oats, shellfish, soy, wheat, peaches, sesame seeds, and olives. His AD was controlled, but not gone, and his EoE was asymptomatic.

The child developed gross hematuria and supra-pubic pain. Urine analysis revealed large blood and protein. Within two days the blood cleared. A complete blood count and complement studies were normal. A renal ultrasound revealed normal kidneys but a markedly thickened balder wall. A cystoscopy and biopsy was performed. The pathology report of H\&E stained sections showed bladder mucosa with portions of reactive urothelial epithelium. The lamina propria is populated by many eosinophils (areas with greater than 100 intramucosal eosinophils per high-power field) with scattered lymphocytes and plasma cells. Intramucosal hemorrhage and pigmented macrophages are present. No acute inflammation, granulomas, or parasites are identified. A repeat esophageal biopsies was performed electively at the time of the cystoscopy (age 7) on swallowed Budesonide (500 QD HS).

The repeat elective esophageal biopsy was normal, with no eosinophils in the proximal or distal esophageal biopsies. To treat the EC we choose to start oral corticosteroids ( $1 \mathrm{mg} / \mathrm{kg} /$ day), Montelukast, and maintain previously utilized antihistamines. Six weeks later he was asymptomatic, with a normal bladder ultrasound, and the oral corticosteroids was tapered to alternate day. At a dose of corticosteroid of $3 \mathrm{mg}$ on alternate days, 6 weeks later, his bladder ultrasound was still normal, and a further taper of the corticosteroids to 1 on alternate over 4 additional weeks still revealed a normal bladder ultrasound. The oral steroid was discontinued with no additional symptoms over the next 6 weeks.

\section{Discussion}

Well-recognized allergic diseases have a complex pathophysiology, which allows for infiltrating eosinophils [3]. The standard for a EOE diagnosis, for example, requires elevated eosinophils in the proximal and/or distal esophagus after 6 weeks of proton pump inhibitor therapy [4]. Asthma, untreated, will demonstrate eosinophils in the lavage fluid with bronchoscopy, a nasal smear in allergic rhinitis show sheets of eosinophils, and chronic atopic dermatitis reveals eosinophil infiltration, and major basic protein staining [3].

A rare condition, based on literature review, is eosinophilic cystitis $[2,5]$. It's not conventionally considered an allergic disease, but the concept of it being associated with food allergen exposure has been discussed, as recently as 2015 [2]. The same manuscript, as well as a 2000 publication, reviews the published literature on EC [2,5].

It would be very unlikely a food macropeptide could transverse normal glomeruli, to deposit in the bladder; but, the systemic exposure (vascular) dissemination of food macropeptides (micropeptides) to the bladder might be conceivable. In regard to our subject, a considerable number of foods had been re-introduced in the 12 months before the development of the eosinophilic cystitis. If persistent peptides of allergic food do reach the bladder, (via the vascular system), the potential for an allergic process to occur and be persistent enough to induce eosinophilic accumulation and symptoms is possible. With, however, the burgeoning population of food allergy and prevalence of food allergy sensitization in children in the United States, there has not been an increase in new EC cases within this century. This is in sharp contrast to the increasing incidence of EoE, which appears to be largely caused by an abnormal immune response to ingested foods, and which is increasing markedly in industrialized countries [1,4,6,7]. A review article on EC from 2000 indicates a low (4.5\%) co-morbidity of eosinophilic enteritis, with a $16 \%$ presence of allergy and a $13 \%$ co- 
Citation: Hopp RJ, Donovan JM (2017) Eosinophilic Cystitis in a 7-Year-Old-Boy with Eosinophilic Esophagitis, Asthma, Food Allergies, and Atopic Dermatitis. J Allergy Ther 8: 251. doi:10.4172/2155-6121.1000251

Page 2 of 2

existence of asthma [5]. These co-morbidities would suggest an atopic pre-disposition for EC development.

In eosinophilic esophagitis, onset has occurred after the start of oral desensitization for a food that previously had caused an allergic reaction [8]. In our report the child had re-ingested numerous foods that had been originally avoided for EoE and/or AD therapy. Unless all re-introduced foods are again re-avoided, there appears little possibility of proving which food that had been re-ingested might be potentially causing the EC. We choose instead to start oral corticosteroids, Montelukast and maintain previously utilized antihistamines.

If EC is truly an allergic disease with exceedingly rare occurrence, the model of EoE increasing prevalence may predict the future for EC and increased cases will become evident.

\section{References}

1. Hopp RJ (2016) Eosinophilic Esophagitis: From Rare to Commonplace: What are the possible Explanations?. EMJ Gatstroenterol 15: 89-95.
2. Mansouri M (2015) Eosinophilic cystitis and interstitial cystitis: may allergy be the reason? J Ped Nephrology 3: 49-57.

3. Metcalf DD, Pawankar R, Ackerman SJ, Akin C, Clayton F, et al. (2016) Biomarkers of the involvement of mast cells, basophils and eosinophils in asthma and allergic diseases. World Allergy Organ J 9:7.

4. Liacouras CA, Furuta GT, Hirano I, Atkins D, Attwood SE, et al. (2011) Eosinophilic esophagitis: updated consensus recommendations for children and adults. J Allergy Clin Immunol 128: 3-20.

5. Van den Ouden D (2000) Diagnois and management of eosinophilic cystitis. A pooled analysis of 135 cases. Eur Urol 37: 386-394.

6. Platts-Mills TA, Schuyler AJ, Erwin EA, Commins SP, Woodfolk JA, et al. (2016) IgE in the diagnosis and treatment of allergic disease. J Allergy Clin Immunol 137: 1662-1670.

7. Hopp RJ (2012) Eosinophilic Esophagitis in Pediatrics: The Worst of all Possible Allergy Worlds?. J Allergy 2012: 179658.

8. Lucendo AJ, Arias A, Tenias JM (2014) Relation between eosinophilic esophagitis and oral immunotherapy for food allergy: a systematic review with meta-analysis. Ann Allergy Asthma Immunol 113: 624-629. 